\title{
The use of aprotinin in children undergoing operative repair of isolated atrial septal defects
}

\author{
S. DEVI CHIRAVURI MD, TERRI VOEPEL-LEWIS MSN RN, \\ ERIC J. DEVANEY MD AND SHOBHA MALVIYA MD \\ Department of Anesthesiology and Surgery, University of Michigan Health Systems, Ann Arbor, \\ MI, USA
}

\section{Summary}

Background: The purpose of this retrospective study was to examine the benefits, risks and costs associated with aprotinin use in children who underwent repair of an atrial septal defect (ASD). The primary aim was to determine whether the transfusion rate is lower in children who received aprotinin compared with those who did not during ASD repair. The use of aprotinin has been shown to reduce transfusion requirements for children undergoing primary or secondary repair of congenital cardiac anomalies. However, past studies have not reported the benefits of this agent during low complexity procedures such as ASD repair.

Methods: All children who underwent ASD repair over 6 years (3 years pre- and postroutine use of aprotinin for all CPB cases in the institution) were identified, and their medical records reviewed.

Children with multiple congenital cardiac lesions were excluded. The following data were recorded: demographics and baseline laboratory findings, intraoperative use of aprotinin, cardiopulmonary bypass information including details of ultrafiltration, all intraoperative and postoperative transfusions, postoperative bleeding and relevant laboratory findings.

Results: One hundred and fifteen children were included, 66 of whom received aprotinin. Transfusion rates were not different between children who received aprotinin $[n=8(12 \%)]$ and those who did not [ $n=3(6 \%)$ ]. Furthermore, changes in hematocrit were not different between groups. These findings were similar when children $\leq 15 \mathrm{~kg}$ were compared with those $>15 \mathrm{~kg}$.

Conclusions: This study suggests that aprotinin use offers no benefit for children undergoing isolated repair of an ASD.

Keywords: aprotinin; children; atrial septal defect; cardiopulmonary bypass; transfusion

Correspondence to: Dr S. Devi Chiravuri, MD, Department of Anesthesiology, University of Michigan Health Systems, F3900 C. S. Mott Children's Hospital, 1500 E. Medical Center Drive, Ann Arbor, MI 48109-5211, USA (email: devich@umich.edu).

\section{Introduction}

The use of the serine protease inhibitor aprotinin, which inhibits fibrinolysis and preserves platelet function, has been shown to reduce bleeding and 
transfusion requirements in adults and children undergoing cardiopulmonary bypass (CPB) (1-3). A recent meta-analysis of randomized-controlled trials of aprotinin in children found a 33\% reduction in the proportion of children receiving packed red blood cells (PRBCs) or whole blood transfusions following cardiac surgery and a $56 \%$ reduction in the volume of transfused PRBCs in children undergoing primary sternotomy (1). These benefits have led to the increased popularity of aprotinin during pediatric cardiac surgery, particularly in neonates with complex congenital lesions who may be at risk for greater blood loss. In certain populations, however, these benefits may be offset by the potential risks associated with aprotinin including thrombotic complications and allergic reactions, as well as its cost. Recent studies in adults found that aprotinin was a predictive factor for postoperative renal dysfunction and failure, as well as mortality (4-6). These findings are concerning given the widespread use of aprotinin; however, similar data are unavailable in children.

Given the purported benefits of aprotinin, it is routinely administered to most children undergoing $\mathrm{CPB}$ for repair of congenital heart defects in our institution, including those undergoing repair of isolated atrial septal defects (ASD). Despite some evidence suggesting a benefit of aprotinin in children undergoing primary sternotomy (1), there are few data that have evaluated the risk or cost/benefit of aprotinin use in this population of children The purpose of this retrospective review, therefore, was to examine the benefits, risks and costs associated with aprotinin use in children who underwent ASD repair in a single, tertiary care center.

\section{Methods}

This retrospective medical record review was undertaken following approval and waiver of consent from the Institutional Review Board at the University of Michigan. All children who underwent repair of an ostium secundum ASD with or without partial anomalous pulmonary venous return between January 2000 and January 2006 were identified from the pediatric cardiothoracic surgery and perfusion databases. Children with multiple congenital cardiac lesions were excluded. It was during 2003 that institutional practice changed from routine aprotinin use for only complex congenital heart cases or repeat cardiac surgery to standard use for nearly all cases involving $\mathrm{CPB}$. The decision to use aprotinin during individual cases was made jointly by the anesthesiologist and cardiovascular surgeon. A standard dosing regimen based on the child's body surface area (BSA) was used. This included a 1-ml test dose of aprotinin, followed by a loading dose equivalent to $171.5 \mathrm{ml} \cdot \mathrm{m}^{2}$ BSA with an equal dose added to the CPB prime solution. An infusion of $40 \mathrm{ml} \cdot \mathrm{m}^{2} \mathrm{BSA} \cdot \mathrm{h}^{-1}$ was maintained throughout the case, and discontinued at the time of skin closure in all patients. This dosing regimen is equivalent to the full Hammersmith dosing, commonly referred to as 'high dose'. A standardized protocol for the selection of CPB circuit components based on patient weight, as well as prime solution additives, was in place throughout the study period. Per routine practice, a minimum of $150 \mathrm{ml}$ of PRBCs were added to the CPB prime of all children $\leq 5 \mathrm{~kg}$ and for those $>5 \mathrm{~kg}$ if the resultant hematocrit after hemodilution was calculated to be $\leq 25-30 \%$.

Per standard institutional practice, intraoperative pain management for all patients consisted of a fentanyl bolus $\left(20-40 \mu \mathrm{g} \cdot \mathrm{kg}^{-1}\right)$ followed by a morphine infusion at $30 \mu \mathrm{g} \cdot \mathrm{kg}^{-1} \mathrm{~h}^{-1}$. Children were extubated at the end of surgery or within $4 \mathrm{~h}$ postoperatively. Postoperative pain was managed per routine practice with morphine continuous infusions and bolus dosing as needed via patientcontrolled analgesia in children over 7 years and nurse-controlled analgesia in younger children.

The medical records of children were reviewed and the following data were recorded: patient demographics, medical history including baseline laboratory values, perioperative data including fluids and blood products administered, medications including aprotinin use, details of ultrafiltration and modified ultrafiltration (MUF), durations of CPB, aortic cross clamp, surgery and anesthesia, as well as postoperative outcomes including blood loss (i.e. chest tube drainage), blood product replacement, urine output and laboratory values. Duration of intubation, stay in the intensive care unit (ICU) and hospital as well as the nature and severity of all adverse events were documented.

Data were analyzed using SPSS ${ }^{\mathrm{TM}} 13.0$ for Windows (Chicago, IL, USA). Nonparametric data such as the prevalence of children who had transfusions were analyzed with chi-squared and Fisher's exact tests wherever appropriate. Parametric data including age, 
duration of surgery, blood loss and hematocrit were analyzed using unpaired $t$-tests. $P<0.05$ was accepted as statistically significant.

\section{Results}

One-hundred and fifteen children underwent a secundum ASD repair during the review period, 66 of whom received aprotinin intraoperative. A description of the sample is presented in Table 1.

Table 1

Description of the sample [data presented as $n(\%)$ or mean $\pm \mathrm{SD}$ where applicable]

\begin{tabular}{|c|c|c|}
\hline & $\begin{array}{l}\text { Aprotinin } \\
(\mathrm{n}=66)\end{array}$ & $\begin{array}{c}\text { No aprotinin } \\
(\mathrm{n}=49)\end{array}$ \\
\hline Age (years) & $5.4 \pm 4.2$ & $7.4 \pm 5.5^{*}$ \\
\hline Male gender $(\%)$ & $23(36)$ & $16(33)$ \\
\hline Weight & $21.2 \pm 16.5$ & $28.2 \pm 21.0$ \\
\hline Body surface area $\left(\mathrm{m}^{2}\right)$ & $0.77 \pm 0.36$ & $0.93 \pm 0.48$ \\
\hline Preoperative hematocrit & $37 \pm 3.1$ & $36.5 \pm 3.2$ \\
\hline \multicolumn{3}{|l|}{ Procedure (\%) } \\
\hline ASD secundum & $50(76)$ & $43(88)$ \\
\hline ASD with PDA & $6(9)$ & $2(4)$ \\
\hline ASD with PAPVR & $10(15)$ & $4(8)$ \\
\hline Sinus venosus & $n=2$ & $n=4$ \\
\hline \multicolumn{3}{|l|}{ Baseline values } \\
\hline Blood urea nitrogen $\left(\mathrm{mg} \cdot \mathrm{dl}^{-1}\right)$ & $12.7 \pm 3.8$ & $11.3 \pm 3.2$ \\
\hline Creatinine $\left(\mathrm{mg} \cdot \mathrm{dl}^{-1}\right)$ & $0.49 \pm 0.11$ & $0.53 \pm 0.17$ \\
\hline Clamp time (min) & $20.6 \pm 7.9$ & $18.4 \pm 8.8$ \\
\hline $\mathrm{CPB}$ duration (min) & $48.3 \pm 12.9$ & $47.4 \pm 14.4$ \\
\hline Ultrafiltrate removed (MUF) $\mathrm{ml} \cdot \mathrm{kg}^{-1}$ & $27.6 \pm 31.5$ & $21.0 \pm 28.2$ \\
\hline
\end{tabular}

ASD, atrial septal defect; PDA, patent ductus arteriosus; PAPVR, partial anomalous pulmonary venous return; $\mathrm{CPB}$, cardiopulmonary bypass; MUF, modified ultrafiltration.

${ }^{*} P=0.038$ compared with aprotinin group.
Children who received aprotinin were significantly younger than controls, but were otherwise similar in demographics and in intraoperative characteristics. Table 2 presents the clinical outcomes in the study groups. There were no differences between groups in the prevalence of intraoperative transfusion, postoperative hematocrit levels, changes in hematocrit from baseline, postoperative bleeding and transfusion rates or in length of stay.

Table 3 presents a comparison of CPB factors, aprotinin use, and outcomes in children $\leq$ and $>15 \mathrm{~kg}$, as the use of MUF is always used in the smaller children in this setting, and rarely in those over this cutoff. There were no differences in hematocrit or transfusion rates in children who received aprotinin compared to those who did not in either weight group. There were no changes from baseline in kidney function tests (i.e. blood urea nitrogen and creatinine levels) for either group of children (see Table 2), and only one child (aprotinin group) experienced a postoperative adverse event (i.e. phrenic nerve palsy) which prolonged the hospital course.

The cost of aprotinin use was calculated for this sample based on a unit cost of $\$ 202.39$ per $100 \mathrm{ml}$ vial. One to six vials of aprotinin were used per patient, and the total cost ranged from $\$ 340$ to $\$ 800$ (mean \$570 \pm 237 ) per case.

\section{Discussion}

This retrospective review of 115 children who underwent an isolated ASD repair found no differences in
Table 2

Clinical outcomes in the study groups

\begin{tabular}{|c|c|c|}
\hline & Aprotinin $(\mathrm{n}=66)$ & No aprotinin $(\mathrm{n}=49)$ \\
\hline Packed RBC in CPB circuit $n(\%)$ & $11(17)$ & $10(20)$ \\
\hline \multicolumn{3}{|l|}{ Hematocrit ( $\%$ change from baseline) } \\
\hline Post-CPB & $29.9 \pm 6.6(-18.6 \pm 19.4)$ & $31.1 \pm 6.0(-14.3 \pm 17.2)$ \\
\hline $4 \mathrm{~h}$ & $31.7 \pm 6.8(-13.8 \pm 19.7)$ & $32.5 \pm 5.7(-10.3 \pm 16.9)$ \\
\hline $24 \mathrm{~h}$ & $30.6 \pm 5.3(-17.2 \pm 15.8)$ & $30.0 \pm 4.7(-16.8 \pm 14.9)$ \\
\hline 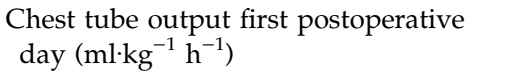 & $0.5 \pm 0.3$ & $0.5 \pm 0.3$ \\
\hline Postoperative transfusion $n(\%)$ & $8(12)$ & $3(6)$ \\
\hline Packed RBC $\left(\mathrm{ml} \cdot \mathrm{kg}^{-1}\right)$ & $16.8 \pm 7.3(n=5)$ & $8.8(n=1)$ \\
\hline Platelets $\left(\mathrm{ml} \cdot \mathrm{kg}^{-1}\right)$ & $8.6(n=1)$ & $9.9(n=1)$ \\
\hline $\mathrm{FFP}\left(\mathrm{ml} \cdot \mathrm{kg}^{-1}\right)$ & $9.1 \pm 0.28(n=2)$ & $10.5(n=1)$ \\
\hline \multicolumn{3}{|l|}{ Postoperative laboratory values } \\
\hline Blood urea nitrogen $\left(\mathrm{mg} \cdot \mathrm{dl}^{-1}\right)$ & $0.4 \pm 0.12$ & $9.8 \pm 2.9$ \\
\hline Creatinine $\left(\mathrm{mg} \cdot \mathrm{dl}^{-1}\right)$ & $9.7 \pm 2.9$ & $0.5 \pm 0.17$ \\
\hline Intensive care unit length of stay (days) & $1.2 \pm 0.8$ & $1.1 \pm 0.6$ \\
\hline Hospital length of stay (days) & $3.8 \pm 1.6$ & $3.6 \pm 0.7$ \\
\hline
\end{tabular}

RBC, red blood cells; FFP, fresh frozen plasma. 
Table 3

Outcomes by weight classification

\begin{tabular}{|c|c|c|c|c|}
\hline & \multicolumn{2}{|c|}{$\leq 15 \mathrm{~kg}(\mathrm{n}=51)$} & \multicolumn{2}{|c|}{$>15 \mathrm{~kg}(\mathrm{n}=64)$} \\
\hline & Aprotinin $(\mathrm{n}=34)$ & No aprotinin $(\mathrm{n}=17)$ & Aprotinin $(\mathrm{n}=32)$ & No aprotinin $(\mathrm{n}=32)$ \\
\hline Ultrafiltration $\left(\mathrm{ml} \cdot \mathrm{kg}^{-1}\right)$ & $48.9 \pm 28.7(n=30)$ & $54.4 \pm 17.9(n=17)$ & $34.7 \pm 14.6(n=4)$ & $30.4 \pm 2.4(n=3)$ \\
\hline \multicolumn{5}{|l|}{ Hematocrit ( $\%$ change) } \\
\hline Post-CPB & $-18.0 \pm 24.2$ & $-11.9 \pm 22.3$ & $-19.6 \pm 12.8$ & $-15.6 \pm 14.0$ \\
\hline $4 \mathrm{~h}$ & $-15.9 \pm 24.1$ & $-9.1 \pm 21.6$ & $-11.7 \pm 13.6$ & $-11.0 \pm 14.1$ \\
\hline \multicolumn{5}{|c|}{ Chest tube output $\left(\mathrm{ml} \cdot \mathrm{kg}^{-1} \mathrm{~h}^{-1}\right)$} \\
\hline $0-4 \mathrm{~h}$ postoperatively & $1.38 \pm 0.97$ & $1.72 \pm 0.097$ & $0.62 \pm 0.48$ & $1.1 \pm 0.7^{* *}$ \\
\hline $4-8 \mathrm{~h}$ postoperatively & $0.74 \pm 0.76$ & $0.8 \pm 0.92$ & $0.32 \pm 0.38$ & $0.58 \pm 0.45^{* *}$ \\
\hline $0-24 \mathrm{~h}$ postoperatively & $0.7 \pm 0.3$ & $0.7 \pm 0.3$ & $0.4 \pm 0.2$ & $0.5 \pm 0.3^{* *}$ \\
\hline Transfused $n(\%)$ & $5(15)$ & $2(12)$ & $3(9)$ & $1(6)$ \\
\hline
\end{tabular}

$* * P=0.03$ compared with aprotinin in $>15 \mathrm{~kg}$ group.

MUF, modified ultrafiltration.

intraoperative transfusion rates, postoperative blood loss, or changes in hematocrit among children who received aprotinin and those who did not. Although blood loss was statistically and significantly higher in children $>15 \mathrm{~kg}$ who did not receive aprotinin compared with children of similar weight who received the agent, the transfusion rates in these groups were not different. These findings suggest that aprotinin use offers no benefit for most children undergoing primary and isolated repair of ASD.

Several previous studies have evaluated the use of aprotinin in the pediatric population and yielded mixed findings (7-9), However, a recent meta-analysis which included all randomized-controlled pediatric trials found that, overall, aprotinin use reduced the proportion of children requiring transfusion of red blood cells or whole blood by 33\% (1). The studies included in this analysis involved over 600 children who had undergone primary or secondary sternotomy. Further analysis of these data showed a 56\% reduction in the proportion of children undergoing primary sternotomy who required transfusion. Findings from the present study, conversely, found no difference in the proportion of children requiring transfusion between the aprotinin and no aprotinin groups. This difference may be explained, in part, by the greater complexity of procedures included in previous studies (e.g. tetralogy of Fallot, aortic arch repair, valve replacement and others). It remains unclear whether any children included in previous studies underwent repair of an isolated $\operatorname{ASD}(7,9,10)$. Additionally, the average duration of $\mathrm{CPB}$ in previous studies was substantially longer than in the present study (65-136 compared with 48 min, respectively), further emphasizing the differences in surgical complexity of the study samples. Differences in the findings therefore suggest that aprotinin offers greater benefit during complex repairs but little or no benefit for low complexity procedures requiring short bypass times and few postoperative transfusions.

Past studies have evaluated the effects of aprotinin in infants smaller than $10 \mathrm{~kg}$ and demonstrated reductions in the number of those requiring transfusions following aprotinin use (1). Smaller infants tend to undergo more complex procedures, may have coagulation defects (11), and experience greater hemodilution during CPB. These factors all increase the likelihood of transfusion following CPB. In this study, we separately evaluated infants $<15 \mathrm{~kg}$ as, in our institution, the $\mathrm{CPB}$ circuitry is standardized for this weight group, and all of these children undergo MUF following CPB to remove excess crystalloid with resultant hemoconcentration of the child's circulating blood. This process itself decreases postoperative transfusion requirements (12). When we analyzed smaller (i.e. $\leq 15 \mathrm{~kg}$ ) and larger children (i.e. $>15 \mathrm{~kg}$ ) separately, the use of aprotinin was associated with reduced blood loss only in children $>15 \mathrm{~kg}$ (see Table 3). Despite this difference in blood loss, the rate of transfusion was similar in children who received aprotinin compared with those who did not. Importantly, the sample of children in these subgroups was very small which may have reduced the power to detect significant differences in these outcomes, and the possibility of a beta error cannot be dismissed. Additionally, it remains unclear from 
our data whether certain subsets of children such as those with bleeding tendencies may benefit from the use of aprotinin even for noncomplex primary surgery including ASD repair. For such children, the decision to use aprotinin should be individualized on a case-by-case basis.

In their meta-analysis, Arnold et al. (1) demonstrated no differences in chest tube drainage or in volume of blood transfused postoperatively in any subgroup of children, suggesting little if any sustained benefit for aprotinin use. These investigators suggested that the transfusion-sparing effect of aprotinin should be weighed against associated risks and costs. While previous studies in children demonstrated no significant differences in adverse events following aprotinin use, recent studies in adults have raised concerns regarding the potential associations between aprotinin use and thrombotic events including stroke and myocardial infarction, as well as renal dysfunction and failure (4-6). Such findings have heightened clinician concerns regarding the riskbenefit profile of this drug and its appropriate use in practice. While previous randomized studies reported no incidences of thrombotic events in children, one case of superficial thrombosis was reported in a 14-year-old child with acute promyelocytic leukemia and a coagulation disorder after administration of aprotinin. The risk of venous thrombosis and stroke in high-risk populations of children should not be disregarded as such complications tend to be poorly captured in clinical trials (1).

There is also a significant risk of hypersensitivity and anaphylactic reactions to aprotinin as it is a polypeptide (13). The reported overall risk of anaphylaxis in adults is $1.8 \%$; however, re-exposure to the drug increases this risk, with the greatest risk (5\% incidence) in patients following re-exposure within 6 months of initial treatment (14). One such case involved an infant undergoing elective replacement of the right ventricular pulmonary artery conduit following repair of tetralogy of Fallot as a neonate. This child experienced an immediate decrease in blood pressure and a sudden increase in peak airway pressure following the test dose of aprotinin [10 000 kallikrein inactivator units (KIU)], and subsequent laboratory analysis found significantly increased aprotinin-specific immunoglobulin E (15). Such reports suggest that aprotinin should be used judiciously in infants and young children, perhaps reserving its use for subsequent surgery when a higher risk of blood loss is anticipated.

The retrospective nature of this study poses several limitations to the ability to generalize these findings. Firstly, despite the change in institutional practice to the routine use of aprotinin for all children undergoing $\mathrm{CPB}$ which occurred half way through the study period, there remains the possibility of a selection bias in decisions to use or not use this agent. Additionally, while CPB protocols including standard prime solutions, circuitry and use of MUF did not vary over this timeframe, variability in clinical decision making may have influenced the outcomes in unknown ways. Further prospective study of aprotinin use in children undergoing specific procedures may be warranted.

This study compared outcomes in children who received aprotinin to those who did not for primary repair of an ostium secundum ASD during the past 6 years in one institution. Aprotinin was not associated with reduced requirements for postoperative blood transfusions in this population, suggesting that use of this agent offers no benefit to children undergoing repair of an isolated ASD.

\section{References}

1 Arnold DM, Fergusson DA, Chan AK et al. Avoiding transfusions in children undergoing cardiac surgery: a meta-analysis of randomized trials of aprotinin. Anesth Analg 2006; 102: 731-737.

2 Henry DA, Moxey AJ, Carless PA et al. Anti-fibrinolytic use for minimising perioperative allogeneic blood transfusion. Cochrane Database Syst Rev 3, 2007.

3 Sedrakyan A, Treasure T, Elefteriades JA. Effect of aprotinin on clinical outcomes in coronary artery bypass graft surgery: a systematic review and meta-analysis of randomized clinical trials. J Thorac Cardiovasc Surg 2004; 128: 442-448.

4 Mangano DT, Tudor IC, Dietzel C et al. The risk associated with aprotinin in cardiac surgery. N Engl J Med 2006; 354: 353365.

5 Karkouti K, Beattie WS, Dattilo KM et al. A propensity score case-control comparison of aprotinin and tranexamic acid in high-transfusion-risk cardiac surgery. Transfusion 2006; 46: 327-338.

6 Mangano DT, Miao Y, Vuylsteke A et al. Mortality associated with aprotinin during 5 years following coronary artery bypass graft surgery. JAMA 2007; 297: 471-479.

7 Boldt J, Knothe C, Zickmann B et al. Comparison of two aprotinin dosage regimens in pediatric patients having cardiac operations. Influence on platelet function and blood loss (see comment). J Thorac Cardiovasc Surgery 1993; 105: 705-711.

8 D'Errico CC, Shayevitz JR, Martindale SJ et al. The efficacy and cost of aprotinin in children undergoing reoperative open heart surgery. Anesth Analg 1996; 83: 1193-1199. 
9 Herynkopf F, Lucchese F, Pereira E et al. Aprotinin in children undergoing correction of congenital heart defects. A doubleblind pilot study. J Thorac Cardiovasc Surg 1994; 108: 517-521.

10 Mossinger H, Dietrich W, Braun SL et al. High-dose aprotinin reduces activation of hemostasis, allogeneic blood requirement, and duration of postoperative ventilation in pediatric cardiac surgery. Ann Thorac Surg 2003; 75: 430-437.

11 Kern FH, Morana NJ, Sears JJ et al. Coagulation defects in neonates during cardiopulmonary bypass. Ann Thorac Surg 1992; 54: 541-546.

12 Naik SK, Knight A, Elliott MJ. A successful modification of ultrafiltration for cardiopulmonary bypass in children. Perfusion 1991; 6: 41-650.
13 Levy JH. Hemostatic agents and their safety. J Cardiothorac Vasc Anesth 1999; 13: 6-11.

14 Dietrich W. Incidence of hypersensitivity reactions. Ann Thorac Surg 1998; 65: S60-S64.

15 Cohen DM, Norberto J, Cartabuke R et al. Severe anaphylactic reaction after primary exposure to aprotinin. Ann Thorac Surg 1999; 67: 837-838.

Accepted 16 July 2007 